\title{
On the Notion of the Measure of Inertia in the Special Relativity Theory
}

\author{
Sergey A. Vasiliev ${ }^{1}$ \\ ${ }^{1}$ Scientific Research Institute of Exploration Geophysics VNIIGeofizika (retired), Moscow 107140, Russia \\ Correspondence: Sergey A. Vasiliev, 38 Nazliu Str., Palio Faliro, Athens 17564, Greece. Tel: 30-210-948-0145. \\ E-mail: disput22@gmail.com,disput22@mail.ru
}

Received: November 11, 2011

Accepted: December 8, 2011 Online Published: May 1, 2012

doi:10.5539/apr.v4n2p136

URL: http://dx.doi.org/10.5539/apr.v4n2p136

\begin{abstract}
The concept of the relativistic mass and its equivalence to the energy was recently negated within the framework of the special relativity theory (SRT). As a consequence, the relativistic mass notion was excluded from majority modern textbooks and books on SRT. The analysis of this negation is carried out in this paper. By the definition, the mass is the measure of inertia. Therefore everywhere, where inertia exists, the measure of this inertia, that is the mass, should exist. The inertia exists at relativistic velocities. Hence, the relativistic mass is obliged to be presented in SRT. The founders of SRT were right in their formulations from the very beginning and there is no need to revise their physical approaches concerning the relativistic measure of inertia. At the correct approach to the problem, the relativistic mass is returned to SRT. The relativistic mass is the measure of inertia, but it is not a scalar in SRT. It is the component of a 4-vector here. Since the relativistic mass is the component of the 4-vector, the fundamental equivalence of the measure of inertia and the energy is valid at all velocities (less or equal to the light velocity). The above mentioned negation is not harmless for science because it closes the road to some basic researches and generates the confusion in the students' brains.
\end{abstract}

Keywords: relativistic mass, measure of inertia, special relativity theory, 4-vector, mass-energy equivalence, Okun', notion

\section{The Aim of the Paper}

In the special relativity theory (SRT) the Einstein's mass-energy relationship

$$
m=\frac{E}{c^{2}}
$$

began to be subjected to the negation afterwards. In it $m$ is the relativistic mass of a physical body, $E$ is its energy, $c$ is the light velocity. Most sequentially and fully the Concept of the opponents is expressed in the papers (Okun', 1989, 2000).

According to this Concept (Okun', 1989, 2000):

1) The relation (1) is not real and has no physical sense because the relativistic mass has no physical sense (I shall speak simply "mass" in follow-up);

2) Only the relation

$$
m_{0}=\frac{E_{0}}{c^{2}}
$$

between a rest-mass $m_{0}$ and rest energy $E_{0}$ has the physical sense;

3) The relativistic mass does not play the role of the measure of inertia and of the gravitational mass;

4) From all masses, only the rest-mass is the invariant of the Lorentz transformation. The rest-mass of the system of not interacting, freely flying particles depends on the angles between the momenta of the particles. In particular (Okun', 2000), the paradox of the photon system takes place: the rest-mass "of a pair of photons, each having energy $E$, is $2 E / c^{2}$ if they move in opposite directions and vanishes if they propagate in the same direction. This is difficult to comprehend for an inexperienced reader who has never before dealt with the theory of relativity, but this is an established fact!". 
Within the framework of this Concept $1-4$, the item 4 is methodologically associated with the items 1-3. According to (Okun', 2000), the formula (1) "was frequently applied to a massless photon as well, muddling the students' brains to utter confusion (on the one hand, the photon is massless; on the other hand, it has a mass)". The Concept 1 - 4 was widely spread up to the exceptions of a relativistic mass from the manuals of physics in Russia (under L.B. Okun's insisting (Okun', 1989, 2000)). According to the review (Wong \& Yap, 2005), the same has taken place in a number of other countries. The majority of the modern books on SRT adhere to this Concept $1-4$, but the alternate concepts on the energy-mass relationship are also existing (Wong \& Yap, 2005).

Meanwhile, the Concept $1-4$ is not so firm, as it seems. Therefore it is necessary to state the critical estimation of the Concept $1-4$. The purpose of the present paper is to show, that all items $1-4$ of the Concept are annulated, if at the deduction of these items to not infringe the requirement of a correctness that should promote elimination of the above muddle and the confusion from the students' brains. Everywhere further, for brevity, we shall term as small velocities, the velocities, much less than the light velocity, and as relativistic velocities - all other velocities, less or equal $c$.

\section{The Principle of Existence of the Mass and the Requirement of Correctness}

Almost all depends on how to understand the term "mass". By the definition, the mass is a measure of inertia. Just so the mass is considered in the present paper. Therefore we shall formulate the principle of existence of a mass: everywhere, where a inertia exists, the measure of this inertia, that is the mass, should exists. At relativistic velocities the inertia exists. Hence, the relativistic mass should exist. For understanding of the inaccuracy of the Concept $1-4$, the following has the decisive importance: at the transition from the classical to the relativistic mechanics, it is impossible to require a priori: 1 ) that the measure of inertia - the mass - is a scalar; 2) that the vector of force $\boldsymbol{F}$ and the vector of acceleration $\boldsymbol{a}$, caused by this force, are parallel; 3 ) that the mass was determined by the ratio of the module of the force $\boldsymbol{F}$ to the module of the acceleration $\boldsymbol{a}$. The given requirements are not imposed a priori also in the classical mechanics, but follow there from a set of experiments, generalized by the Newton's second law of motion. But the classical mechanics "knows nothing" that really happens at the relativistic velocities, i.e. outside of the applicability of the classical mechanics. Hence, it is impossible to transfer a priori the above conditions from the classical mechanics to the relativistic mechanics. At the relativistic velocities in order to decide correctly the problems on the scalaricy or nonscalaricy of masses, on the parallelism or no parallelism of the vectors $\boldsymbol{F}$ and $\boldsymbol{a}$, on the sense of the ration of the modules of the force and the acceleration, it is necessary to be based or on the direct measurings of the physical quantities (in particular, $\boldsymbol{F}$ and $\boldsymbol{a}$ ) at relativistic velocities, or on the equations of SRT, following of other direct experiments (for example, on the fixing of the constancy of the light velocity), but the problems to not solve in any way a priori. Many other things are impossible to transfer a priori from the classical to the relativistic mechanics, for example, the Newton's second law. In the classical mechanics, the Newton's second law and the masses scalaricy are cleanly the experimental facts, besides not having any explanation. So why the Newton's second law is not transferred a priori from the classical to the relativistic mechanics, but it is modified by known manner, and the masses scalaricy is transferred a priori sometimes? This does not have a reasonable explanation. The above prohibitions for the a priori transferences we shall term as the requirements of correctness.

From the told clearly: as inertia exists at the relativistic velocities the relativistic mass is obliged to exist. And what will be a relativistic mass - a scalar, a vector, a tensor - depends on transformations of the Newton's laws and some other relations at an exit out the limits of applicability of the classical mechanics. May be, the masses nonscalaricy is not blended in some theories. But, if the masses nonscalaricy will follow from the experiments or from the equations basing on experiments, the author is predisposed to give the priority to the Nature. In fact only so the physics can come nearer to the truth.

At the substantiation of the Concept $1-4$, all the above requirements of correctness are infringed. At the attempts of the definition of the relativistic mass, a priori the mass is supposed with a scalar (Okun', 1989, 2000) (NOTE 1), and, for the definition of the mass in the relativistic mechanics, the Newton's second law from the classical mechanics was used (Okun', 1989, 2000), that is simply the module of force is divided by the module of the acceleration (hence, it is a priori was considered, that the mass has sense only at the parallelism of the vectors $\boldsymbol{F}$ and $\boldsymbol{a})$. As a result of the infringements of the requirements of a correctness, the deductions about senselessness of the relativistic mass, about injustice of the fundamental law $E=m c^{2}$ and about validity of all items 1 - 4 have been obtained. Supporters of the Concept $1-4$, obviously, have come in the contradiction with the principle of the mass existence. Moreover, the paradox of the photon system has arisen as an artefact, i. e. as the result of the not entirely correct building-up of the paradox (this is in detail the lower). Quite the contrary: the more paradoxes of the physical sense arise at the negation of the relativistic mass existence. 


\section{The Paradoxes of the Concept 1 - 4}

The concept 1 - 4 lead to the following inconsistent no adequacies of the physical sense. In accordance with the Concept $1-4$, the progressing electromagnetic waves and photons have no any mass. But for reflection of the photon it is required to transmit the momentum to the photon. Signifies, photons have the inertia. Then the following arises: the inertia is, and the measure of the inertia is not, the gravitational action on the photon is, and the gravitational mass of the photon is not. And for any particle with a nonzero rest-mass $m_{0}$ there is the same inadequacy: the particle has the inertia of the motion, but, according to the concept $1-4$, has no the measure of this inertia during the motion. Or, as is known (Landau \& Lifschitz, 1967), the mass of the physical body consisting of the particles, is incremented at the increase of the velocities of the particles, i. e. the kinetic energy of the particles contributes to the measure of the inertia of the physical body, but, according to the Concept $1-4$, for one reason the kinetic energy does not contribute to the measure of the inertia of the particles. The list of the no adequacies, arising from the Concept $1-4$, is possible to prolong.

Einstein was right from the very outset. In present paper the relativistic definition of the measure of inertia is advanced. In result, the paradox of the system of photons is eliminated; the strangenesses of the physical sense and the infringements of the principle of the existence of a mass disappear. At the relativistic definition of the notion of the inertia measure, the relativistic mass $m$ play role of the inertia measure and of the measure of the gravitational mass at any velocities (smaller or equal $c$ ). Energies of the motion of the particles contributes in their masses. The electromagnetic waves and photons have the relativistic mass, the gravitational field acts them according to their gravitational mass, that is equivalent their inertia measure.

\section{On the Existence of the Relativistic Mass}

The purpose of a relativistic mechanics is generalization of the rules of the classical mechanics on the case of the relativistic velocities. If sequentially to be guided by this purpose, and by virtue of the principle of the existence of a mass, in SRT it is necessary to generalize the classical notion of the inertia measure on the case of the relativistic velocities. Therefore the relativistic mass appears an essential part of SRT. Hence, it is necessary to search for the relativistic measure of inertia instead of to be focused to examinations of extremely that, the ration of the modules of the force and the acceleration is whether or not the relativistic mass. The searching of the relativistic measure of inertia with the help of division of the module of the force on the module of the acceleration has not lead to success (Okun', 1989, 2000). Hence, it is necessary to search for the inertia measure in SRT by some other way. At that it is not required to devise new formulas of SRT. It is enough to use the known equations of SRT, resulting of the direct experiments and the relativity principle. The inertia is appeared in two interrelations: 1) in the interrelation of the force $\mathbf{F}$ and the acceleration $\boldsymbol{a} ; 2$ ) in the interrelation of the momentum $\mathbf{p}$ and the velocity $\mathbf{v}$ (NOTE 2). The kernel of the problem consist not in that, the result of the division of the module of the force on the module of the acceleration is whether or not the inertia measure in SRT, that is explored in works (Okun', 1989, 2000). The kernel of the problem consist in the searching of such the inertia measure which unambiguously determinates the acceleration $\boldsymbol{a}$ at the given force $\mathbf{F}$, or the momentum $\mathbf{p}$ - at the given velocity $\mathbf{v}$. To find the concrete form of the relativistic inertia measure - the relativistic mass $m$ it is enough to define it by the rule (1) and to show, that the defined by such a way relativistic mass is really the inertia measure.

Let's be convinced, that defined by the given a way the relativistic mass $m$ of a particle, at first, is the measure of its inertia in SRT, secondly, is equivalent to its gravitational mass in SRT, thirdly, follows to the equality

$$
m=\gamma m_{0} \quad \text { при } \quad m_{0}>0,
$$

entered for the relativistic mass by the founders SRT, where

$$
\gamma \equiv \frac{1}{\sqrt{1-\beta^{2}}}, \quad \boldsymbol{\beta} \equiv \frac{\mathbf{v}}{c}, \quad \beta \equiv|\boldsymbol{\beta}|=\frac{\mathrm{v}}{c},
$$

$\mathbf{v}$ - the vector of the velocity of the particle, $\quad \mathbf{v} \equiv|\mathbf{v}|$.

Really, the equations of the relativistic mechanics for the free particle has form [3]

$$
E^{2}-c^{2} \mathbf{p}^{2}=m_{0}^{2} c^{4},
$$




$$
\mathbf{p}=\frac{E}{c^{2}} \mathbf{v}
$$

Here $\mathbf{p}$ - the vector of the momentum of the particle. These equations are true and at the zero rest-mass $m_{0}$.

Inertia of the particle is exhibited, in particular, by the transmission to the particle of momentum. At that the role of the inertia measure of the particle is played by the multiplier that is situated in expression (6) before velocity $\mathbf{v}$ (NOTE 3). The multiplier in expression (6) is magnitude $E / c^{2}$. Hence, the definition (1) really expresses the inertia measure in the relativistic mechanics. The substitution (6) in (5) gives a relation

$$
E^{2}\left(1-\beta^{2}\right)=m_{0}^{2} c^{4}
$$

and by virtue of the definition (1) we obtain the formula (3). Besides contrary to the Concept 1 - 4, by virtue of the formula (1), the interrelationship of the relativistic measure of inertia and the energy $\left(E=m c^{2}\right)$ appears valid both at small, and at relativistic velocities as it was required to show concerning on the inertial mass. The energy is the component of the energy - momentum 4-vector. Hence, and the relativistic mass is not a scalar, but is the component of the 4-vector that corresponds to the above requirements of a correctness (NOTE 4). Strictly speaking, and at the small velocities, the relativistic mass $m$ remains the vector component, but varies so feebly, that is practically indistinguishable from the scalar $m_{0}$. Using the reasons from the book (Landau and Lifschitz, 1967), we come to the conclusion about validity of the made developments and for a composite physical body (Landau and Lifschitz, 1967): "Let's underline, that though we speak here about a "particle", but its "simplicity" is not used anywhere. Therefore the obtained formulas are equally applicable and to any composite physical body consisting of many particles, and under a mass $m_{0}$ it is necessary to understand the complete rest-mass of the physical body, and as the velocity - the velocity of its motion as the whole." - the end of the citation.

\section{On the Infringements of the Requirements of a Correctness}

In work (Okun', 2000) it is a priori posited: "mass is a relativistic scalar while energy is a 4-vector component". This is the first infringement of the requirements of correctness.

Further, in view of formulas (3), (4), the Newton's second law of the classical mechanics

$$
\frac{\mathbf{F}}{m_{0}}=\frac{d \mathbf{v}}{d t} \equiv \mathbf{a}
$$

is transformed in SRT to the form (Landau \& Lifschitz, 1967; Okun', 1989)

or, that is the same,

$$
\mathbf{F}=\frac{d \mathbf{p}}{d t}=\frac{d(m \mathbf{v})}{d t}=\gamma m_{0} \mathbf{a}+\gamma^{3} m_{0}(\boldsymbol{\beta}, \mathbf{a}) \boldsymbol{\beta},
$$

$$
\mathbf{F}-(\mathbf{F}, \boldsymbol{\beta}) \boldsymbol{\beta}=\gamma m_{0} \mathbf{a},
$$

where the parentheses with the comma in the middle mean a scalar product of vectors. According to paper (Okun', 1989): "Despite the unusual appearance of Eq. (9) from the point of view of Newtonian mechanics (we should say, rather, precisely because of this unusual appearance), this equation correctly describes the motion of relativistic particles. From the beginning of the century it was frequently submitted to experimental verification for different configurations of the electric and magnetic fields. This equation is the foundation of the engineering calculations for relativistic accelerators. Thus, if $\mathbf{F} \perp \mathbf{v}$, then $\mathbf{F}=\gamma m_{0} \mathbf{a}$, but if $\mathbf{F} / / \mathbf{v}$, then $\mathbf{F}=\gamma^{3} m_{0} \mathbf{a}$. Thus, if one attempts to define an "inertial mass" as the ration of the force to the acceleration, then in the theory of relativity this quantity depends on the direction of the force relative to the velocity, and therefore cannot be unambiguously defined." - the end of the citation. Evidently, within the framework of the Concept $1-4$, for the definition of the relativistic inertia measure it is used a priori the no relativistic Newton's law (8) - the mass a priori is determined by the division of the force magnitude on the acceleration magnitude. It is the second, the most essential, infringement of the requirements of correctness.

From the above replacement of the relativistic Newton's law (9) by its no relativistic analog (8) at the attempts of the definition of the notion of the relativistic inertia measure, follows the negation of the relativistic inertia measure, the contradiction with the principle of existence of a mass and the principle inaccurate conclusions described in the items $1-4$ of the first section (please, see lower about a gravitational mass). From the above 
citations of the papers (Okun', 1989, 2000) it is well visible, that the searching of the relativistic inertia measure in the frame of the Concept $1-4$ is realized a priori so, as if at the exit out of the limits of the classical mechanics validity there is no expansions of physical notions (as if outside of the indicated limits, as before, only the ratio of the modules of the force and acceleration should unambiguously to determinate the mass).

\section{On the Relativistic Mass as the Inertia Measure in the Relativistic Generalization of the Newton's second Law}

Taking into account the definition (1), the equation (9) permit its rewriting in the shape similar to the classical Newton's second law,

$$
\frac{\mathbf{F}}{m}=\mathbf{W}(\boldsymbol{\beta} ; \mathbf{a})
$$

where

$$
\mathbf{W}(\boldsymbol{\beta} ; \mathbf{a}) \equiv \mathbf{a}+\gamma^{2}(\boldsymbol{\beta}, \mathbf{a}) \boldsymbol{\beta} .
$$

Thus, though the Newton's second law varies at the passage to relativistic velocities, but its general structure is remained: the left-hand parts of the equations (8), (11) is the result of division of the force on the magnitude $m_{0}$ or $m$, and the right members of these equations does not depend neither from $\mathbf{F}$, nor from $m_{0}$ or $m$. In the classical mechanics the ratio $\boldsymbol{F} / m_{0}$ unambiguously determinates the acceleration $\boldsymbol{a}$ of the particle through the classical Newton's laws (8), strictly speaking, only at the zero velocity of the particle. In the relativistic mechanics the ratio $\boldsymbol{F} / m$ unambiguously determinates the acceleration $\boldsymbol{a}$ at the given both zero, and any nonzero velocity $\mathbf{V}$ of the particle (smaller $c$ ) through the relativistic Newton's laws (11). Therefore determinate by equality (1) the relativistic mass $m$ is the inertia measure including and from the point of view of the interdependence of the force $\boldsymbol{F}$ and the acceleration $\boldsymbol{a}$ induced by this force. Laws of the Nature are dilated at the transition from the classical to the relativistic mechanics. Therefore it is quite natural that, in contrast to the classical mechanics, in SRT the acceleration depends not only on the ratio of the force and the inertia measure, but else depends on the velocity of the particle, due to the contribution of the term $\gamma^{2}(\boldsymbol{\beta}, \boldsymbol{\alpha}) \boldsymbol{\beta}$ in equality (12).

Thus the rule of the parallelism of the force and acceleration is violated - the acceleration has the component parallel to the force, but besides acquires the component parallel to the velocity $\mathbf{v}$ of the particle that it is easy to see taking into account the contribution of the item $(\mathbf{F}, \boldsymbol{\beta}) \boldsymbol{\beta}$ to the formula (10). It happens as though the "leeway" of the acceleration in the direction of the velocity. The physical sense of the last is known and clear. The last is natural since Lorentz transformations do not change the spatial coordinates perpendicular to the velocity, but change only one spatial coordinate, namely - parallel to the velocity. In this sense there is the selected direction (the direction of the velocity) to which the acceleration has "leeway".

\section{On the Mass - energy Equivalence}

As it mentioned above, the formula $E=m c^{2}$ of the interdependence of a mass and an energy, is valid in SRT at any velocities (smaller or equal $c$ ). According to paper (Okun', 1989): "the formula $E=m$ (I again set $c=1$ ) is ugly, since it represents an extremely unfortunate designation of the energy $E$ by a further letter and term, these being, moreover, the letter and term associated in physics with another important concept. The only justification of the formula is the historical justification - at the beginning of the century it helped the creators of the theory of relativity to create this theory. From the historical point of view, this formula and everything associated with it can be regarded as the remnants of the scaffolding used in the construction of the beautiful edifice of modern science. But to judge from the literature, it is today regarded as almost the principal portal of this edifice." Probably, the formula $m=E / c^{2}$ can seem ugly, but only as long as it is merely the denotation. To tell the truth, at that remains not clear why, according to paper (Okun', 1989), the relation $m_{0}=E_{0} / c^{2}$ is not ugly. In fact, with the magnitude $m_{0}$, as before, is "associated in physics with another important concept", than the notion of the energy $E_{0}$. However, starting with the moment when it is shown, that the magnitude $m$ is the inertia measure and the 4-vector component, the formula $m=E / c^{2}$ is not ugly, and expresses the fundamental physical law: the energy (which, in the final analysis, is the ability to make work) unambiguously determinates the inertia measure, and vice versa. Starting with this moment, the above literature is right: the formula $m=E / c^{2}$ really represents the principal portal of SRT. When it will be understood, why the physically dissimilar features - the energy $E$ and the inertia measure $m$ - appear one-to-one coupled, the new horizon of the understanding of a physical reality will open. Seemingly, this law means, in essence, that there is a certain common physical substance which generates both the property of matter to have the energy and the property of mater to have the inertia. If it is so, to reject the indicated law means to reject a fundamental direction of physical examinations and to exclude from examinations the essential physical properties. Then the Concept $1-4$ is not harmless for the science. 


\section{On the Item 4 of the Concept 1 - 4}

It is easy to see, that, at the correct definition (1), the relativistic mass of the system of the free particles (perhaps and photons) always is equal to the sum of the relativistic masses of the particles, irrespectively of the directions of the particles' momenta and the paradox of the photons' system is automatically excluded. It follows immediately from the definition (1) if to take into account, that the energy of the system of the free particles is equal to the sum of the energies of these particles (NOTE 5).

According to item 4 of the Concept 1 - 4, from all masses, only the rest-mass is an invariant of Lorentz transformation. Let $\mathbf{v}_{0}$ is the zero velocity of some particle $\left(\mathbf{v}_{0}=0\right)$, and $\mathbf{v}_{1}$ is anyone, but the fixed velocity of this particle, $\left|\mathbf{v}_{1}\right|<c$. The relativistic mass of the particle $m$ depends on its velocity $\mathbf{v}$ by the rule (3). In other words, the magnitude $m$ is function of the velocity $\mathbf{v}, m=m(\mathbf{v})$. In any inertial reference frame $S$, on the definition, the rest-mass $m_{0}$ is the relativistic mass $m\left(\mathbf{v}_{0}\right)$ at the fixed, zero velocity $\mathbf{v}_{0}$ particle, $m_{0} \equiv m\left(\mathbf{v}_{0}\right)$. In any inertial reference frame $S$, we shall define the mass $m_{1}$ at anyone, but the fixed velocity $\mathbf{v}_{1}$ by the rule $m_{1} \equiv m\left(\mathbf{v}_{1}\right)$. If in each system $S$ forcedly to stop the particle then in all the systems $S$ the mass of the particle will be identical and equal to a rest-mass of a particle $m_{0} \equiv m\left(\mathbf{v}_{0}\right)$. Only in such respect, the rest-mass $m_{0}$ is invariant of Lorentz transformation. But, evidently, if in each system $S$ forcedly to impel the particle to move with anyone, but the same velocity $\mathbf{v}_{1}$ then in all systems $S$ the mass of the particle will be again identical, and it is equal to a mass $m_{1}$, $m_{1} \equiv m\left(\mathbf{v}_{1}\right)$. Hence, any mass $m_{1}$ at anyone, but the fixed velocity $\mathbf{v}_{1}$ is the same invariant of Lorentz transformation, as well as the rest-mass $m_{0}$ taken at the fixed zero velocity. Hence, the masses $m_{0}$ and $m_{1}$ are equivalent in the special relativity theory. (But they are the conditional invariants of Lorentz transformation, i.e. the invariance of the mass is observed under the condition of "obtrusion" to the particle of the same fixed velocity in different inertial reference frames. If to the particle to impose nothing and simply to observe it from the point of view of different inertial reference frames $S$ the mass is not a scalar, but is the 4-vector component in relation to Lorentz transformation.)

\section{On the Equivalence of an Inertial and Gravitational Masses}

Let there is a motion of the particle of the mass $m$ (for example an electron or a photon) under the influence of a constant gravitational field of the heavy, not shifted body of a mass $M_{S}$, i.e. $M_{S} \gg m$. Then, at the passage from the classical to relativistic mechanics, the classical Newton's gravitation law

$$
\mathbf{F}=-\frac{m_{0} G M_{S} \mathbf{r}}{|\mathbf{r}|^{3}}
$$

it is transformed to the form (Okun', 1989):

$$
\mathbf{F}=-\frac{G M_{S} m\left[\left(1+\beta^{2}\right) \mathbf{r}-(\mathbf{r}, \boldsymbol{\beta}) \boldsymbol{\beta}\right]}{|\mathbf{r}|^{3}},
$$

where $m=E / c^{2}$ - the relativistic inertial mass of the particle with an energy $E ; \mathbf{F}$ - the force of gravitational action on the particle; $\mathbf{r}$ - the radius-vector joining the particle and the source of the field, directional from the source; $G$ - the gravitational constant; $\mathbf{v}$ - the velocity of the particle. According to work (Okun', 1989): "Whereas in Newtonian theory the force of the gravitational interaction is determined by the masses of the interacting bodies, in the relativistic case the situation is much more complicated. It is case to see that for a slow electron, with $\beta<<1$, the expression in the square bracket reduces to $\mathbf{r}$, and, bearing in mind that $E_{0} / c^{2}=m_{0}$, we return to Newton's nonrelativistic formula. However, for $\beta \approx 1$ or $\beta=1$ we encounter a fundamentally new phenomenon, namely, the quantity that plays the role of the "gravitational mass" of the relativistic particle depends not only on this energy but also on the mutual direction of the vectors $\mathbf{r}$ and $\mathbf{v}$. If $\mathbf{v} / / \mathbf{r}$, then the "gravitational mass" is $E / c^{2}$, but is $\mathbf{v} \perp \mathbf{r}$, it is $\left(E / c^{2}\right)\left(1+\beta^{2}\right)$, and for a photon $2 E / c^{2}$. We use the quotation marks to emphasize that for a relativistic body the concept of gravitational mass of a photon this quantity is half that for one traveling horizontally." - the end of the citation. In the quoted Position again as earlier concerning an inertial mass, for definition of the relativistic gravitational mass of the particle the nonrelativistic law of gravitation (13) is used and by that the requirements of the correctness are violated. However, the role of a relativistic gravitational mass consist not at all to define uniquely the gravitational force through the nonrelativistic law of gravitation, but in to define uniquely the gravitational force through the relativistic law of gravitation.

The relativistic formula (14) is reduced to the classical formula (13) only, when $\beta=0$. So, the classical relation (13) correctly defines the gravitational force $\boldsymbol{F}$, strictly speaking, only at the zero velocity of the particle. Let the 
radius-vector $\mathbf{r}$ is anyone, but the fixed. Then in the classical mechanics the inertial mass $m_{0}$ unambiguously and correctly defines the gravitational force $\boldsymbol{F}$, strictly speaking, only at the zero velocity of the particle through the classical Newton's law of gravity (13). Therefore the inertial mass $m_{0}$ is equivalent to the gravitational mass, strictly speaking, only at the zero velocity of the particle. In the relativistic mechanics the inertial relativistic mass $m$ unambiguously and correctly defines the gravitational force $\boldsymbol{F}$ at the given both zero, and any nonzero velocity $\mathbf{v}$ of the particle ( $\mathbf{v}$ is smaller $c$ ) through the relativistic variant of the Newton's law of gravity (14). Therefore the given by equation (1) relativistic inertial mass $m$ is the gravitational mass at any velocities $\mathbf{v}$ of the particle (smaller $c$ ). The law of gravity is varied at the passage from the classical to relativistic mechanics. Therefore it is quite natural, that, as against to the classical mechanics, in SRT the gravitational force $\boldsymbol{F}$ depends not only on the gravitational mass of the particle, but else depends on the velocity of the particle. Due to adding of the term $\beta^{2} \mathbf{r}-(\mathbf{r}, \boldsymbol{\beta}) \boldsymbol{\beta}$ in the equation (14), the gravitational force obtains the component, parallel to the velocity of the particle, that is again happens the "leeway", but now not of the acceleration, and of the gravitational force. According to experience of the development of physics, similar modifications of physical laws of the classical physics, at an exit out of the limits of its applicability, are quite natural. There is no sense to try "to pen" the adding $\beta^{2} \mathbf{r}-(\mathbf{r}, \boldsymbol{\beta}) \boldsymbol{\beta}$ into the gravitational mass.

\section{Resume}

According to the principle of existence of the mass, the relativistic measure of inertia should be presented in the special relativity theory (SRT). But the mass is not obliged to remain a scalar at the passage from the classical to relativistic mechanics. We have demonstrated that the relativistic measure of inertia, i.e. the relativistic mass, exists in SRT and it is not a scalar quantity but it is the component of the 4-vector. The relativistic mass plays the role of the measure of inertia in the interrelation between the force and acceleration caused by this force as well as in the interrelation between the momentum and the velocity. At that any mass $m_{1}$ at anyone, but the fixed velocity $\mathbf{v}_{1}$, is the same invariant of Lorentz transformation as well as the rest-mass $m_{0}$ taken at the fixed zero velocity (please, look above section 8). The use of the relativistic mass in SRT is sequential fulfillment of the purpose of SRT and is inalienable of SRT. Since the relativistic mass is the component of the 4-vector, the fundamental interrelation $E=m c^{2}$ between the measure of inertia and the energy is correct at any velocity (less or equal $c$ ). Probably, it means, that there is a certain common physical substance which generates both the property of matter to have energy, and the property of matter to have inertia. It is possible to show, that the relativistic inertial mass plays in SRT the role of the relativistic gravitational mass, at least, at constant gravitational field.

The opposite conclusions of the supporters of the Concept 1 - 4 (section 1), are obtained as result of the violation of the requirements of a correctness. At the correct analysis: all items 1-4 of the Concept 1-4 are vanished; the relativistic mass of the system of not interacting, freely flying particles is equal always to the sum of the relativistic masses of particles and does not depend on the angles between the momenta of the particles; the paradox of the photons system does not arise. The concept $1-4$ is not harmless for a science since this Concept closes the road to the investigations of the physical reasons of the mass-energy $E=m c^{2}$ equivalence, to the investigations of the reasons of the inertia variations at the variations of the physical body motions and to study of the contiguous problems.

It is stated sometimes, that it is useless to introduce the relativistic mass $m$ because by the definition (1) the relativistic mass $m$ is simply other measure of the energy. However, at first, it is not the strong argument, at least, as long as it is not told: it is useless to introduce the rest-mass $m_{0}$ too since by virtue of the fundamental relation (2), $m_{0}$ is other measure of the energy too, but now of the rest energy. Secondly, the kernel of the problem is not in that to introduce or to not introduce the designation $m$. The kernel of the problem is in that the relativistic measure of inertia is necessary in SRT, and in that the energy appears to be the measure of this inertia.

Unfortunately, the majority of the modern books on SRT adhere to the Concept 1-4 (Wong and Kueh, 2005). Therefore the inexact and distorted representation on the special relativity theory can arise among the students, if educational process to not supplement with similar explanations.

The author is grateful to professor D. D. Rabounski and professor I. V. Veselovskiy for contansive discussions.

\section{Notes}

Note 1. It could be understood somehow still if the mass was a measure not of inertia but some measure of the amount of the material invariant relatively Lorentz transforms. 
Note 2. The more inertia of the physical body - the variation of its velocity should be less at the delivery of the predetermined momentum to the physical body.

Note 3. In fact, the more this multiplier, the more the inertia of the particle, that is, the less the particle velocity variation at the transmission to the particle of the fixed momentum.

Note 4. In principle, the researcher could define the relativistic measure of inertia ambiguously. For example, any function $f(m)$ from a mass $m$ could serve as a measure of inertia if function $f(m)$ approach to $m_{0}$ when the velocity $\mathbf{v}$ approach to zero and if between function $f(m)$ and the mass $m$ there is an one-to-one correspondence. The same ambiguity takes place and in the classical mechanics. But, hardly, it is convenient to use other measure of inertia rather than magnitude $m$.

Note 5. Applying here the formula (1) to the analysis of the system of the free particles, we obtain the result formally. In order that the formal result has acquired the physical sense, it is necessary from the beginning to have defined the notion of the motion of the system of the independent particles, as the single whole. (Differently it is not clear, in relation to what acceleration the inertia of the independent particles system is discussed when speak on the mass of the independent particles system.) For this purpose it is possible to enter the special system of coordinates relative to which the physical properties of the free particles (their masses, velocities, momenta, energies) remain constants - "frozen" in this system of coordinates - but which can acquire acceleration. Such "frozen" population of the coordinates and the particles, appropriate to the chosen set of the particles, the author terms as the appropriate system, or briefly as $\underline{a-s y s t e m}$. Then acceleration of the system of the free particles (perhaps and photons) acquire the sense, as the acceleration of their a-system. The summarized relativistic mass of the particles appears the rest-mass of their a-system. The acceleration of the a-system of a photon gives rise to changing not of the velocity of the photon, but to changing of its energy. Applications of the a-systems and the discrepancies of the construction of the apparent paradox of the photons system (Okun', 2000) are in details considered in the work (Vasiliev, 2009).

\section{References}

Landau, L. D., \& Lifschitz, E. M. (1967). The Theory of Fields. Moscow, Nauka, 458.

Okun', L. B. (1989). The concept of mass. Usp. Fiz. Nauk, 158, 511-530. (in Russian). (Sov. Phys. Usp., 32, 629-638, 1989, in English)

Okun', L. B. (2000). Reply to the letter "What is mass?" by R I Khrapko. Usp. Fiz. Nauk, 170, 1366-1371. (in Russian). (Sov. Phys. Usp., 43, 1270-1275, 2000, in English)

Vasiliev, S. A. (2009). On the role of the relativistic mass in the special relativity theory. The Earth Planet System, Proceedings of XVII-th Scientific Seminar, 15 years to the interdisciplinary scientific seminar, Moscow State University, Moscow, The monography. Moscow, LENAND, p.105-116. (in Russian). Retrieved from http://www.nonmaterial.pochta.ru or http://www.nonmaterial.narod.ru

Wong, Chee Leong, \& Yap, Kueh Chin. (2005), Conceptual Development of Einstein's Mass-Energy Relationship. New Horizons in Education, 51, May, 56-66. Retrieved from http://www.eric.ed.gov/PDFS/EJ848449.pdf 